九州大学学術情報リポジトリ

Kyushu University Institutional Repository

Non-existence of certain Galois representations with a uniform tame inertia weight

Ozeki, Yoshiyasu

The JSPS Fellowships for Young Scientists

http://hdl. handle. net/2324/16842

出版情報 : MI Preprint Series. 2010-14，2010-03-25. 九州大学大学院数理学研究院 バージョン：

権利関係 : 


\section{Preprint Series}

Kyushu University

The Global COE Program

Math-for-Industry Education \& Research Hub

\section{Non-existence of certain Galois representations with a uniform tame inertia weight}

\section{Yoshiyasu Ozeki}

MI 2010-14

( Received March 25, 2010 )

Faculty of Mathematics

Kyushu University

Fukuoka, JAPAN 


\title{
Non-existence of certain Galois representations with a uniform tame inertia weight
}

\author{
Yoshiyasu Ozeki*
}

\begin{abstract}
In this paper, we prove the non-existence of certain semistable Galois representations of a number field. Our consequence can be applied to some geometric problems. For example, we prove a special case of a Conjecture of Rasmussen and Tamagawa, related with the finiteness of the set of isomorphism classes of abelian varieties with constrained prime power torsion.
\end{abstract}

\section{Introduction}

Let $\ell$ be a prime number and $K$ a number field. In this paper, we show the non-existence of certain semistable $\ell$-adic Galois representations of the absolute Galois group $G_{K}$ of $K$ by using remarkable results on the tame inertia weights due to Caruso. Fix non-negative integers $n, r$ and $w$, and a prime number $\ell_{0} \neq \ell$. Put $\bullet:=\left(n, \ell_{0}, r, w\right)$. We consider the set $\operatorname{Rep}_{\mathbb{Q}_{\ell}}\left(G_{K}\right)^{\bullet}$ of isomorphism classes of $\ell$-adic representations of $G_{K}$ (Definition $2.4(2)$ ). This set is related with the dual of $H_{\text {ét }}^{w}\left(X_{\bar{K}}, \mathbb{Q}_{\ell}\right)$, where $X$ is a proper smooth scheme over $K$ which has everywhere semistable reduction and has good reduction at a place of $K$ above $\ell_{0}$. Our main result in this paper is

Theorem 0.1 (= Theorem 2.11). Suppose that $w$ is odd or $w>2 r$. Then there exists an explicit constant $C$ depending only on $K, n, \ell_{0}, r$ and $w$ such that $\operatorname{Rep}_{\mathbb{Q}_{\ell}}\left(G_{K}\right)^{\bullet}$ is empty for any prime number $\ell>C$ which does not split in $K$.

Theorem 0.1 comes from a relation between the tame inertia weights and eigenvalues of Frobenius action (Proposition 2.8). As a by-product of the above theorem, we obtain some approaches to algebraic geometry. For example, our result gives an application to a special case of the RasmussenTamagawa conjecture $([\mathrm{RT}])$ related with the finiteness of the set of isomorphism classes of abelian varieties with constrained prime power torsion.

Now we describe an organization of this paper. In Section 1, we recall some results on integral $p$-adic Hodge theory given by Caruso [Ca]. In Section 2, we give explicit values of the tame inertia weights for certain semistable Galois representations and prove our non-existence theorem. In Section 3, we apply our consequence for some geometric problems.

Acknowledgements. The author wish to thank Shin Hattori for bringing the work of Xavier Caruso. The author would like to express his sincere gratitude to Akio Tamagawa and Seidai Yasuda who pointed out the mistake of the previous version of the proof for the main theorem and gave him useful advise.

\section{Notation:}

For a prime number $\ell$ and a topological group $G$, an $\ell$-adic representation of $G$ (resp. $\mathbb{F}_{\ell}$-representation) is a finite-dimensional $\mathbb{Q}_{\ell}$-vector space $V$ (resp. $\mathbb{F}_{\ell}$-vector space $V$ ) equipped with a continuous and linear $G$-action. For such a representation $V$, we denote by $V^{\vee}$ the dual of $V$, that is,

* Supported by the JSPS Fellowships for Young Scientists.

e-mail: y-ozeki@math.kyushu-u.ac.jp 
$V^{\vee}:=\operatorname{Hom}_{\mathbb{Q}_{\ell}}\left(V, \mathbb{Q}_{\ell}\right)\left(\right.$ resp. $\left.V^{\vee}:=\operatorname{Hom}_{\mathbb{F}_{\ell}}\left(V, \mathbb{F}_{\ell}\right)\right)$ with the $G$-action defined by $g . f(v):=f\left(g^{-1} \cdot v\right)$ for $f \in V^{\vee}, g \in G$ and $v \in V$. For any scheme $X$ over a commutative ring $R$ and an $R$-algebra $R^{\prime}$, we denote the fiber product $X \times_{\operatorname{Spec}(R)} \operatorname{Spec}\left(R^{\prime}\right)$ by $X_{R^{\prime}}$.

\section{Tame inertia weights of semistable representations}

In this section, we recall the definition of the tame inertia weights (cf. [Se], Section 1) and Caruso's work for the tame inertia weights of a residual representation of semistable Galois representations (cf. [Ca]). Let $K_{\lambda}$ be a complete discrete valuation field of characteristic zero with perfect residue field $k$ of positive characteristic $\ell$ and $G_{K_{\lambda}}$ its absolute Galois groups. Let $e$ be the absolute ramification index of $K_{\lambda}$. The tame inertia weights of an $\ell$-adic semistable Galois representation of $G_{K_{\lambda}}$ with Hodge-Tate weights in $[0, r]$ have remarkable properties if $e r<\ell-1$. For example, Serre conjectured in [Se] that the tame inertia weights on the Jordan-Hölder quotients of a residual representation of the $r$-th $\ell$-adic étale cohomology group $H_{\text {ét }}^{r}\left(X_{\bar{K}_{\lambda}}, \mathbb{Q}_{\ell}\right)$ of a proper smooth scheme $X$ over $K_{\lambda}$ are between 0 and er. Caruso proved this Serre's conjecture in [Ca] by using the integral $p$-adic Hodge theory. As an another example, in [CS], Caruso and Savitt proposed the tame inertia polygon of an $\ell$-adic semistable Galois representation of $G_{K_{\lambda}}$, and they showed that this polygon has good relations with the Hodge polygon and the Newton polygon introduced in [Fo].

\subsection{Tame inertia weights}

We denote by $I_{\lambda}$ the inertia subgroup of $G_{K_{\lambda}}, I_{w}$ its wild inertia subgroup and $I_{t}:=I_{\lambda} / I_{w}$ the tame inertia group. Let $V$ be an $h$-dimensional irreducible $\mathbb{F}_{\ell}$-representation of $I_{\lambda}$ and fix a separable closure $\mathbb{F}_{\ell}$ of $\mathbb{F}_{\ell}$. By the irreducibility, the action of $I_{\lambda}$ on $V$ factors through $I_{t}$ and thus we can regard $V$ as a representation of $I_{t}$. Applying Schur's lemma, we see that $\mathbb{E}:=\operatorname{End}_{I_{t}}(V)$ is the finite field of order $\ell^{h}$. Moreover, the representation $V$ inherits a structure of a 1-dimensional $\mathbb{E}$ representation of $I_{t}$ by the natural manner. This representation is given by a character $\rho: I_{t} \rightarrow \mathbb{E}^{\times}$. Choose any isomorphism $f: \mathbb{E} \rightarrow \mathbb{F}_{\ell^{h}}$ and consider the composition $\rho_{f}: I_{t} \stackrel{\rho}{\rightarrow} \mathbb{E}^{\times} \stackrel{f}{\rightarrow} \mathbb{F}_{\ell^{h}}^{\times}$:

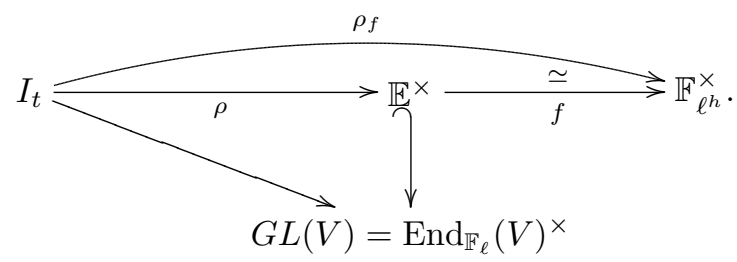

Denote by $\mu_{\ell^{h}-1}\left(\bar{K}_{\lambda}\right)$ the set of $\left(\ell^{h}-1\right)$-st roots of unity in a separable closure $\bar{K}_{\lambda}$ of $K_{\lambda}$. Consider an isomorphism $\mu_{\ell^{h}-1}\left(\bar{K}_{\lambda}\right) \simeq \mathbb{F}_{\ell^{h}}^{\times}$coming from a surjection $\mathcal{O}_{\bar{K}_{\lambda}} \rightarrow \overline{\mathbb{F}}_{\ell}$, where $\mathcal{O}_{\bar{K}_{\lambda}}$ is the integer ring of $\bar{K}_{\lambda}$, and take the following fundamental character of level $h$ :

$$
\begin{aligned}
\theta_{h}: I_{t} & \rightarrow \mu_{q-1}\left(\bar{K}_{\lambda}\right) \simeq \mathbb{F}_{\ell^{h}}^{\times} . \\
\sigma & \mapsto \frac{\eta^{\sigma}}{\eta}
\end{aligned}
$$

Here $\eta$ is a $\left(\ell^{h}-1\right)$-st root of a uniformizer of $K_{\lambda}$. It is easy to check that $\theta_{h}^{1+\ell+\cdots+\ell^{h-1}}=\theta_{1}$, $\theta_{h}^{\ell^{h}-1}=1$ and, with respect to $h$ embeddings $\mathbb{F}_{\ell^{h}} \hookrightarrow \overline{\mathbb{F}}_{\ell}$, all the fundamental characters are given by $\theta_{h, 0}\left(:=\theta_{h}\right), \theta_{h, 1}, \theta_{h, 2}, \ldots, \theta_{h, h-1}$, where $\theta_{h, i}=\theta_{h, i-1}^{\ell}$ for $0 \leq i \leq h-1$ and $\theta_{h, 0}=\theta_{h, h-1}^{\ell}$. It is known that $\theta_{1}^{e}$ coincides with the mod $\ell$ cyclotomic character ([Se], Section 1.8, Proposition 8). Since $I_{t}$ is pro-cyclic and $\operatorname{Im}\left(\theta_{h}\right)=\mathbb{F}_{\ell^{h}}$, there exists an integer $n_{f} \in\left\{0,1, \ldots, \ell^{h}-2\right\}$ such that $\rho_{f}=\theta_{h}^{n_{f}}$. If we decompose $n_{f}=n_{0}+n_{1} \ell+n_{2} \ell^{2}+\cdots+n_{h-1} \ell^{h-1}$ with integers $0 \leq n_{i} \leq \ell-1$ for any $i$, then we can see that the set $\left\{n_{0}, n_{1}, n_{2}, \ldots, n_{h-1}\right\}$ is independent of the choice of $f$.

Definition 1.1. We call these numbers $n_{0}, n_{1}, n_{2}, \ldots, n_{h-1}$ the tame inertia weights of $V$. In general, for any $\mathbb{F}_{\ell}$-representation $V$ of $I_{\lambda}$, the tame inertia weights of $V$ are the numbers of the tame inertia weights of all the Jordan-Hölder quotients of $V$. 
Example 1.2. Suppose that $k$ is algebraically closed. Let $E$ be an elliptic curve over $K_{\lambda}$ with semistable reduction. If $E$ has supersingular reduction, assume $e=1$. Then the tame inertia weights of $E[\ell]$ are 0 and $e$ (cf. [Se], Section 1, Proposition 11 and 12).

Definition 1.3. Let $V$ be an $\ell$-adic representation of $G_{K_{\lambda}}$. The tame inertia weights of $V$ is the tame inertia weights of a residual representation of $\left.V\right|_{I_{\lambda}}$.

The above definition is independent of the choice of a residual representation of $V$ by the Brauer-Nesbitt theorem.

Definition 1.4. Let $w$ be an integer with $0 \leq w<\ell-1$ and $V$ be an $n$-dimensional $\ell$-adic representation of $G_{K_{\lambda}}$. Denote by $w_{1} \leq w_{2} \leq \cdots \leq w_{n}$ all the tame inertia weights of $V$. We say that $V$ is of uniform tame inertia weight $w$ if $w_{1}=w_{2}=\cdots=w_{n}=w$.

\subsection{Caruso's Result}

Fix an integer $r \geq 0$ such that $e r<\ell-1$. We use the ring $S$ and the category $\operatorname{Mod}_{/ S \infty}^{r, \Phi}, N$ of finite torsion $S$-modules equipped with some additional structures as in Section 1 of $[\mathrm{Ca}]$ without giving the precise definitions. The category $\operatorname{Mod}_{/ S_{\infty}}^{r, \Phi, N}$ is just the category $\underline{\mathcal{M}}^{r}$ given in op.cit. The category $\operatorname{Mod}_{/ S_{\infty}}^{r, \Phi, N}$ is an abelian category (cf. [Ca], Section 3.5). We denote by $\operatorname{Rep}_{\mathbb{Z}_{\ell}}^{\text {st }}\left(G_{K_{\lambda}}\right)^{r}$ (resp. $\left.\operatorname{Rep}_{\mathbb{Z}_{\ell}}\left(G_{K_{\lambda}}\right)_{\text {tors }}\right)$ the category of $G_{K_{\lambda}}$-stable $\mathbb{Z}_{\ell}$-lattices of semistable $\ell$-adic representations of $G_{K_{\lambda}}$ with Hodge-Tate weights in $[0, r]$ (resp. the category of finite torsion $\mathbb{Z}_{\ell}$-modules with a continuous $G_{K_{\lambda}}$-action). Denote by $\operatorname{Mod}_{/ S}^{r, \Phi, N}$ the category of strongly divisible modules over $S$ of weight $r$ (cf. [Ca], Section 7.1). There exist the following two contravariant functors

$$
T_{\mathrm{st}}: \operatorname{Mod}_{i S}^{r, \Phi, N} \rightarrow \operatorname{Rep}_{\mathbb{Z}_{\ell}}^{\mathrm{st}}\left(G_{K_{\lambda}}\right)^{r}
$$

and

$$
T_{\mathrm{st}}: \operatorname{Mod}_{/ S_{\infty}}^{r, \Phi, N} \rightarrow \operatorname{Rep}_{\mathbb{Z}_{\ell}}\left(G_{K_{\lambda}}\right)_{\text {tors }}
$$

satisfying good properties. For example,

(1) (cf. [Ca], Theorem 1.0.5) The 1st $T_{\mathrm{st}}$ is an isomorphism,

(2) (cf. [Ca], Theorem 1.0.4) The 2 nd $T_{\text {st }}$ is exact and fully faithful, and its essential image is stable under taking sub-objects and quotient objects.

If $\mathcal{M} \in \operatorname{Mod}^{r, \Phi, N}$ is isomorphic to $S / \ell^{n_{1}} S \oplus S / \ell^{n_{2}} S \oplus \cdots \oplus S / \ell^{n_{d}} S$ as $S$-modules, then $T_{\text {st }}(\mathcal{M})$ is

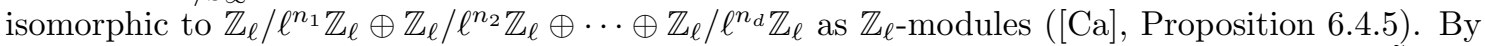
the definition of strongly divisible modules, we see that, for any strongly divisible module $\tilde{\mathcal{M}}$ and $n \geq 0$, the quotient $\tilde{\mathcal{M}} / \ell^{n} \tilde{\mathcal{M}}$ is an object of $\operatorname{Mod}_{/ S_{\infty}}^{r, \Phi, N}$ and the following diagram is commutative:

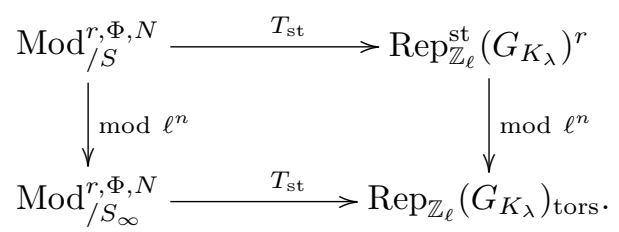

If $k$ is algebraically closed and $\mathcal{M} \in \operatorname{Mod}_{/ S_{\infty}}^{r, \Phi, N}$ is a simple object, then $T_{\text {st }}(\mathcal{M})$ is an irreducible $\mathbb{F}_{\ell}$-representation of $G_{K_{\lambda}}$ and its tame inertia weights are between 0 and $\operatorname{er}$ ([Ca], Theorem 1.0.3). By using the above facts, we can show the following important theorem:

Theorem $1.5([\mathrm{Ca}])$. Let $T_{\ell} \in \operatorname{Rep}_{\mathbb{Z}_{\ell}}^{\mathrm{st}}\left(G_{K_{\lambda}}\right)^{r}$ and $\bar{T}_{\ell}=T_{\ell} / \ell T_{\ell}$ its residual representation. Then the tame inertia weights of $\left.\bar{T}_{\ell}\right|_{I_{\lambda}}$ are between 0 and er. 
Proof. We may assume that $k$ is algebraically closed. Choose the strongly divisible module $\tilde{\mathcal{M}}$ corresponding to $T_{\ell}$ via $T_{\mathrm{st}}$. Then $\mathcal{M}:=\tilde{\mathcal{M}} / \ell \tilde{\mathcal{M}}$ is contained in $\operatorname{Mod}_{/ S_{\infty}}^{r, \Phi}$ and $T_{\mathrm{st}}(\mathcal{M})$ is isomorphic to $\bar{T}_{\ell}$. We identify $T_{\mathrm{st}}(\mathcal{M})$ with $\bar{T}_{\ell}$. Since the essential image of $T_{\mathrm{st}}: \operatorname{Mod}_{/ S_{\infty}}^{r, \Phi, N} \rightarrow \operatorname{Rep}_{\mathbb{Z}_{\ell}}\left(G_{K_{\lambda}}\right)_{\text {tors }}$ is stable under sub-quotient, any Jordan-Hölder quotient of $\bar{T}_{\ell}$ is isomorphic to the representation of the form $T_{\mathrm{st}}\left(\mathcal{M}^{\prime}\right)$ for some $\mathcal{M}^{\prime} \in \operatorname{Mod}_{/ S_{\infty}}^{r, \Phi}$. The object $\mathcal{M}^{\prime}$ is simple because the functor $T_{\mathrm{st}}$ is exact and fully faithful. Therefore, we obtain the desired result.

Remark 1.6. In fact, we do not need the assumption er $<\ell-1$ for Theorem 1.5 (the case $e r \geq \ell-1$ is trivial).

\section{Non-existence theorems}

In this section, we calculate the tame inertia weights of $\ell$-adic representations with certain geometric and filtration conditions for a prime number $\ell$ large enough. As a result, we show the non-existence theorems of certain Galois representations.

Let $K$ be a finite extension over $\mathbb{Q}$ and fix an algebraic closure $\bar{K}$ of $K$. We put $G_{K}:=$ $\operatorname{Gal}(\bar{K} / K)$, the absolute Galois group of $K$. Let $\ell$ be a prime number. For any finite place $v$ of $K$, we denote by $G_{v}$ and $I_{v}$ the decomposition group and its inertia subgroup at $v$, respectively. Furthermore, we denote by $e_{v}$ the absolute ramification index at $v, q_{v}$ the order of the residue field of $v$ and $\operatorname{Fr}_{v}$ the arithmetic Frobenius at $v$. For a place $\lambda$ of $K$ above $\ell$, we identify $G_{\lambda}$ with the absolute Galois group $G_{K_{\lambda}}$ of a $\lambda$-adic completion $K_{\lambda}$ of $K$ via a fixed embedding $\bar{K} \hookrightarrow \bar{K}_{\lambda}$, where $\bar{K}_{\lambda}$ is an algebraic closure of $K_{\lambda}$.

Definition 2.1. Let $\lambda$ be a place of $K$ above $\ell$ and $V$ an $\ell$-adic representation of $G_{K}$. The tame inertia weights of $V$ at $\lambda$ is the tame inertia weights of $\left.V\right|_{G_{\lambda}}$ (cf. Definition 1.3). For an integer $0 \leq w<\ell-1$, we say that $V$ is of uniform tame inertia weight $w$ at $\lambda$ if $\left.V\right|_{G_{\lambda}}$ is of uniform tame inertia weight $w$ (cf. Definition 1.4).

\subsection{Geometric and filtration conditions}

We define the set of representations we mainly consider throughout this section. We fix nonnegative integers $n, r, w$ and $\bar{w}$, and a prime number $\ell_{0}$ different from $\ell$. Let $\chi_{\ell}$ be the $\bmod \ell$ cyclotomic character. Take an $n$-dimensional $\ell$-adic representation $V$ of $G_{K}$ and denote by $\bar{V}$ its residual representation. Now we consider the following geometric conditions (G-1), (G-2), (G-2)' and (G-3), and filtration conditions (F-1) and (F-2):

(G-1) For any place $\lambda$ of $K$ above $\ell$, the representation $\left.V\right|_{G_{\lambda}}$ is semistable and has Hodge-Tate weights in $[0, r]$.

(G-2) For some places $\lambda_{0}$ of $K$ above $\ell_{0}$, the representation $V$ is unramified at $\lambda_{0}$ and the characteristic polynomial $\operatorname{det}\left(T-\operatorname{Fr}_{\lambda_{0}} \mid V\right)$ has rational integer coefficients. Furthermore, there exists nonnegative integers $w_{1}(V), w_{2}(V), \ldots, w_{n}(V)$ such that $w_{1}(V)+w_{2}(V)+\cdots+w_{n}(V) \leq \bar{w}$ and the roots of the above characteristic polynomial have complex absolute values $q_{\lambda_{0}}^{w_{1}(V) / 2}, q_{\lambda_{0}}^{w_{2}(\bar{V}) / 2}, \ldots, q_{\lambda_{0}}^{w_{n}(V) / 2}$ for every embedding $\overline{\mathbb{Q}}_{\ell}$ into $\mathbb{C}$.

$(\mathrm{G}-2)^{\prime}$ The condition (G-2) holds and $w_{1}(V)=w_{2}(V)=\cdots=w_{n}(V)=w$.

(G-3) For any finite place $\lambda$ of $K$ not above $\ell$, the action of $I_{\lambda}$ on $\bar{V}$ is unipotent.

(F-1) The representation $\bar{V}$ has a filtration of $G_{K}$-modules

$$
\{0\}=\bar{V}_{0} \subset \bar{V}_{1} \subset \cdots \subset \bar{V}_{n-1} \subset \bar{V}_{n}=\bar{V}
$$

such that $\bar{V}_{k}$ has dimension $k$ for each $1 \leq k \leq n$. 
(F-2) The condition (F-1) holds. Moreover, for each $1 \leq k \leq n$, the $G_{K}$-action on the quotient $\bar{V}_{k} / \bar{V}_{k-1}$ is given by $g \cdot \bar{v}=\chi_{\ell}^{a_{k}}(g) \bar{v}$ for some $0 \leq a_{k} \leq \ell-2$.

If an $\ell$-adic representation $V$ satisfies the condition (F-1), then we say that $V$ is of residually Borel. We note that it is independent of the choice of a residual representation $\bar{V}$ of $V$ whether the filtration conditions (F-1) and (F-2) hold or not. If $n=2$, then (F-1) is equivalent to the condition that $\bar{V}$ is reducible.

Example 2.2. Suppose $w \leq r$. Let $X$ be a proper smooth scheme over $K$ which has everywhere semistable reduction and has good reduction at some places of $K$ above $\ell_{0}$. Then the dual $H_{\text {ét }}^{w}\left(X_{\bar{K}}, \mathbb{Q}_{\ell}\right)^{\vee}$ of the $w$-th $\ell$-adic étale cohomology group of $X$ satisfies the geometric conditions $(\mathrm{G}-1),(\mathrm{G}-2)^{\prime}$ and $(\mathrm{G}-3)$.

Proposition 2.3. Let $X$ be a proper smooth scheme over $K$ and $w$ an odd integer. Denote by $S_{X}$ the finite set of prime numbers $p$ such that $X$ has bad reduction at some place of $K$ above $p$. Then, there exists a finite extension $L$ of $K$ such that, for any $\ell \notin S_{X}$, the $\ell$-adic representation $H_{\text {ét }}^{w}\left(X_{\bar{L}}, \mathbb{Q}_{\ell}\right)$ of $G_{L}$ is semistable at all finite places.

In particular, we have the following: Let $X$ and $L$ be as above. Fix a prime number $\ell_{0} \notin S_{X}$ and take a prime number $\ell$ such that $\ell \neq \ell_{0}$ and $\ell \notin S_{X}$. Then $H_{\text {ét }}^{w}\left(X_{\bar{L}}, \mathbb{Q}_{\ell}\right)^{\vee}$ satisfies $(\mathrm{G}-1),(\mathrm{G}-2)^{\prime}$ and (G-3) as a representation of $G_{L}$.

Proof of Proposition 2.3. If we admit the semistable conjecture for $X$, then we can prove this proposition easily. However, we can obtain the desired result without using the semistable conjecture as below: For any algebraic extension $K^{\prime}$ of $K$, denote by $S_{X, K^{\prime}}$ the set of places of $K^{\prime}$ which is above one of the prime numbers in $S_{X}$. Take any place $v \in S_{X, K}$. By de Jong's alteration theorem ([dJ], Theorem 6.5), there exist a finite extension $K_{v}^{\prime}$ of $K_{v}$, a proper strictly semistable scheme $\mathcal{Y}^{v}$ over $\mathcal{O}_{K_{v}^{\prime}}$ and a morphism $\mathcal{Y}^{v} \rightarrow \mathcal{X}$ compatible with $\operatorname{Spec}\left(\mathcal{O}_{K_{v}^{\prime}}\right) \rightarrow \operatorname{Spec}\left(\mathcal{O}_{K_{v}}\right)$ such that the morphism $f: \mathcal{Y}^{v} \rightarrow \mathcal{X}_{\mathcal{O}_{K_{v}^{\prime}}}$ induced by the above morphism is an étale alteration (see also [Ts], Theorem A3). Here $\mathcal{X}$ is a proper flat model of $X_{K_{v}}$ over $\mathcal{O}_{K_{v}}$. Such a model always exists by the compactification theorem of Nagata. Take any prime number $\ell^{\prime}$. If we denote by $f_{*}$ and $f^{*}$ the induced homomorphisms $H_{\text {ét }}^{w}\left(\mathcal{Y}_{\bar{K}_{v}^{\prime}}^{v}, \mathbb{Q}_{\ell^{\prime}}\right) \rightarrow H_{\text {ét }}^{w}\left(X_{\bar{K}_{v}}, \mathbb{Q}_{\ell^{\prime}}\right)$ and $H_{\text {ét }}^{w}\left(X_{\bar{K}_{v}}, \mathbb{Q}_{\ell^{\prime}}\right) \rightarrow H_{\text {ét }}^{w}\left(\mathcal{Y}_{\bar{K}_{v}^{\prime}}^{v}, \mathbb{Q}_{\ell^{\prime}}\right)$ respectively, then the map $f_{*} \circ f^{*}$ is the multiplication by $\operatorname{deg}(f)$. In particular, the map $f^{*}$ is injective. Thus we may consider that $H_{\text {ét }}^{w}\left(X_{\bar{K}_{v}^{\prime}}, \mathbb{Q}_{\ell^{\prime}}\right)$ is a sub-representation of $H_{\text {ét }}^{w}\left(\mathcal{Y}_{\bar{K}_{v}^{\prime}}^{v}, \mathbb{Q}_{\ell^{\prime}}\right)$. Now take a finite extension $K(v)$ of $K$ and a place $w(v)$ of $K(v)$ above $v$ such that $K(v)_{w(v)}=K_{v}^{\prime}$, where $K(v)_{w(v)}$ is the $w(v)$-adic completion of $K(v)$. The existence of $K(v)$ and $w(v)$ is an easy consequence of [La], Chapter II, Section 2, Proposition 4. We denote by $L$ the Galois closure, over $K$, of the field generated by all $K(v)$. Here $v$ runs through all the places of $K$ in $S_{X, K}$. Now we take a prime number $\ell \notin S_{X}$. It suffices to show that the $\ell$-adic representation $H_{\text {ét }}^{w}\left(X_{\bar{L}}, \mathbb{Q}_{\ell}\right)$ of $G_{L}$ is everywhere semistable. Take any finite place $w_{L}$ of $L$. If $w_{L} \notin S_{X, L}$, then $X$ has good reduction at $w_{L}$ and in particular $H_{\text {ét }}^{w}\left(X_{\bar{L}}, \mathbb{Q}_{\ell}\right)$ is semistable at $w_{L}$. Suppose $w_{L} \in S_{X, L}$. We denote the restriction of $w_{L}$ to $K$ by $v$. Take $\mathcal{Y}^{v}$ and the place $w(v)$ of $K(v)$ as above. Furthermore, we take a place $w_{L}^{\prime}$ of $L$ above $w(v)$. Since the action of $I_{w_{L}^{\prime}}$ is unipotent on $H_{\text {ét }}^{w}\left(\mathcal{Y}_{L}^{v}, \mathbb{Q}_{\ell}\right)$, we have that the action of $I_{w_{L}^{\prime}}$ on $H_{\text {ét }}^{w}\left(X_{\bar{K}}, \mathbb{Q}_{\ell}\right)$ is unipotent, too. Since the inertia subgroup $I_{w_{L}^{\prime}}$ conjugates with $I_{w_{L}}$ by the element of $G_{K}$, we see that the action of $I_{w_{L}}$ on $H_{\text {ét }}^{w}\left(X_{\bar{K}}, \mathbb{Q}_{\ell}\right)$ is also unipotent, that is, $H_{\text {ét }}^{w}\left(X_{\bar{L}}, \mathbb{Q}_{\ell}\right)$ is semistable at $w_{L}$. This finishes the proof.

Definition 2.4. Put $\circ:=\left(n, \ell_{0}, r, \bar{w}\right)$ and $\bullet:=\left(n, \ell_{0}, r, w\right)$.

(1) We denote by $\operatorname{Rep}_{\mathbb{Q}_{\ell}}\left(G_{K}\right)_{\text {cycl }}^{\circ}\left(\operatorname{resp} \cdot \operatorname{Rep}_{\mathbb{Q}_{\ell}}\left(G_{K}\right)_{\text {cycl }}^{\bullet}\right.$ ) the set of isomorphism classes of $n$ dimensional $\ell$-adic representations $V$ of $G_{K}$ which satisfy $(\mathrm{G}-1),(\mathrm{G}-2)$ and (F-2) (resp. (G-1), $(\mathrm{G}-2)^{\prime}$ and $\left.(\mathrm{F}-2)\right)$.

(2) We denote by $\operatorname{Rep}_{\mathbb{Q}_{\ell}}\left(G_{K}\right)^{\circ}\left(\operatorname{resp} . \operatorname{Rep}_{\mathbb{Q}_{\ell}}\left(G_{K}\right)^{\bullet}\right)$ the set of isomorphism classes of $n$-dimensional $\ell$-adic representations $V$ of $G_{K}$ which satisfy (G-1), (G-2), (G-3) and (F-1) (resp. (G-1), (G-2)', $(\mathrm{G}-3)$ and $(\mathrm{F}-1))$. 
Clearly, we have

$$
\begin{array}{ccc}
\operatorname{Rep}_{\mathbb{Q}_{\ell}}\left(G_{K}\right)_{\mathrm{cycl}}^{\circ} & \subset & \operatorname{Rep}_{\mathbb{Q}_{\ell}}\left(G_{K}\right)^{\circ} \\
\cup & & \cup \\
\operatorname{Rep}_{\mathbb{Q}_{\ell}}\left(G_{K}\right)_{\text {cycl }}^{\bullet} & \subset & \operatorname{Rep}_{\mathbb{Q}_{\ell}}\left(G_{K}\right)^{\bullet},
\end{array}
$$

where $\bullet=\left(n, \ell_{0}, r, w\right)$ and $\circ=\left(n, \ell_{0}, r, \bar{w}\right)$ for any $n w \leq \bar{w}$.

Our main concern in this section is the following question:

Question 2.5. Does there exist a constant $C$ which depends on $K$ and $\bullet($ or $\circ)$ such that the sets defined in Definition 2.4 are empty for $\ell>C$ ? If the answer is positive, how can we evaluate such a constant $C$ ?

Remark 2.6 (Trivial case). Take a representation $V \in \operatorname{Rep}_{\mathbb{Q}_{\ell}}\left(G_{K}\right)^{\bullet}$. By (G-2), the complex absolute value of the determinant of $\mathrm{Fr}_{v_{0}}$ acting on $V$ is $q_{v_{0}}^{n w / 2}$ and this must be an integer. From this fact, if $n$ and $w$ are odd and the extension $K / \mathbb{Q}$ is Galois of an odd degree, then $\operatorname{Rep}_{\mathbb{Q}_{\ell}}\left(G_{K}\right)^{\bullet}$ is empty for any prime $\ell \neq \ell_{0}$. As this example, there exist lots of pairs of $(K, \bullet)(\operatorname{resp}$. $(K, \circ))$ such that $\operatorname{Rep}_{\mathbb{Q}_{\ell}}\left(G_{K}\right)^{\bullet}\left(\right.$ resp. $\left.\operatorname{Rep}_{\mathbb{Q}_{\ell}}\left(G_{K}\right)^{\circ}\right)$ is empty for a prime $\ell$ (large enough). We hope to know "non-trivial cases" of the emptiness of the sets given in Definition 2.4.

\subsection{Main results}

We denote by $d, d_{K}$ and $h_{K}^{+}$the extension degree of $K$ over $\mathbb{Q}$, the discriminant of $K$ and the narrow class number of $K$, respectively. Put $M:=\max \{n r, \bar{w} / 2\}$ and

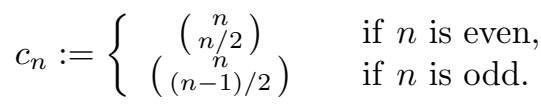

Clearly this is equal to $\max \left\{\left(\begin{array}{c}n \\ m\end{array}\right) \mid 0 \leq m \leq n\right\}$. Now we put

$$
\begin{aligned}
& \varepsilon_{1}:=d M, \quad \varepsilon_{2}:=d \varepsilon_{1}, \quad \varepsilon_{1}^{\prime}:=d h_{K}^{+} M, \quad \varepsilon_{2}^{\prime}:=d \varepsilon_{1}^{\prime}, \\
& C_{1}:=C_{1}(d, \bullet):=2 c_{n} \ell_{0}^{\varepsilon_{1}}, \quad C_{2}:=C_{2}(d, \bullet):=2 c_{n} \ell_{0}^{\varepsilon_{2}}, \\
& C_{1}^{\prime}:=C_{1}^{\prime}(K, \bullet):=2 c_{n} \ell_{0}^{\varepsilon_{1}^{\prime}}, \quad C_{2}^{\prime}:=C_{2}^{\prime}(K, \bullet):=2 c_{n} \ell_{0}^{\varepsilon_{2}^{\prime}} .
\end{aligned}
$$

The following two propositions play an essential role for our main results.

Proposition 2.7. Any $\ell$-adic representation $V$ in the set $\operatorname{Rep}_{\mathbb{Q}_{\ell}}\left(G_{K}\right)_{\text {cycl }}^{\circ}$ has tame inertia weights $e_{\lambda} w_{1}(V) / 2, e_{\lambda} w_{2}(V) / 2, \ldots, e_{\lambda} w_{n}(V) / 2$ at any place $\lambda$ of $K$ above $\ell$ under any one of the following situations:

(a) $\ell \nmid d_{K}$ and $\ell>C_{1}$;

(b) $\ell>C_{2}$.

Proposition 2.8. Suppose that $\ell$ is a prime number which does not split in $K$. Any $\ell$-adic representation $V$ in the set $\operatorname{Rep}_{\mathbb{Q}_{\ell}}\left(G_{K}\right)^{\circ}$ has tame inertia weights $e_{\lambda} w_{1}(V) / 2, e_{\lambda} w_{2}(V) / 2, \ldots, e_{\lambda} w_{n}(V) / 2$ at the unique place $\lambda$ of $K$ above $\ell$ under any one of the following situations:

(a) $\ell \nmid d_{K}$ and $\ell>C_{1}^{\prime}$;

(b) $\ell>C_{2}^{\prime}$.

To prove these propositions, we need the following lemma:

Lemma 2.9. Let $s, t_{1}, t_{2}, \ldots, t_{n}$ and $u$ be non-negative integers such that $0 \leq s \leq u$ and $0 \leq$ $t_{k} \leq r u$ for all $k$. Let $V$ be an $n$-dimensional $\ell$-adic representation of $G_{K}$ which satisfies (G$2)$. Decompose $\operatorname{det}\left(T-\operatorname{Fr}_{\lambda_{0}} \mid V\right)=\prod_{1 \leq k \leq n}\left(T-\alpha_{k}\right)$. If the set $\left\{\alpha_{1}^{s}, \alpha_{2}^{s}, \ldots, \alpha_{n}^{s}\right\}$ coincides with the set $\left\{q_{\lambda_{0}}^{t_{1}}, q_{\lambda_{0}}^{t_{2}}, \ldots, q_{\lambda_{0}}^{t_{n}}\right\}$ in $\overline{\mathbb{F}}_{\ell}$ and $\ell>2 c_{n} \ell_{0}^{d M u}$, then $\left\{\alpha_{1}^{s}, \alpha_{2}^{s}, \ldots, \alpha_{n}^{s}\right\}=\left\{q_{\lambda_{0}}^{t_{1}}, q_{\lambda_{0}}^{t_{2}}, \ldots, q_{\lambda_{0}}^{t_{n}}\right\}$. In particular, we obtain $\left\{s w_{1}(V) / 2, s w_{2}(V) / 2, \ldots, s w_{n}(V) / 2\right\}=\left\{t_{1}, t_{2}, \ldots, t_{n}\right\}$. 
Proof. We basically follow the proof by the method which has been pointed out by Rasmussen and Tamagawa. Let us denote by $S_{m}\left(x_{1}, x_{2}, \ldots, x_{n}\right)$ the elementary symmetric polynomial of degree $m$ with $n$-indeterminates $x_{1}, x_{2}, \ldots, x_{n}$ for $0 \leq m \leq n$, that is,

$$
\prod_{1 \leq k \leq n}\left(T-x_{k}\right)=\sum_{0 \leq m \leq n} S_{m}\left(x_{1}, x_{2}, \ldots, x_{n}\right) T^{n-m} .
$$

For any $0 \leq m \leq n$, the condition (G-2) implies that $S_{m}\left(\alpha_{1}, \alpha_{2}, \ldots, \alpha_{n}\right)$ is a rational integer for all $m$ and hence $S_{m}\left(\alpha_{1}^{s}, \alpha_{2}^{s}, \ldots, \alpha_{n}^{s}\right)$, which is a symmetric polynomial of $\alpha_{1}, \alpha_{2}, \ldots, \alpha_{n}$, is also a rational integer. On the other hand, we have

$$
\begin{aligned}
\left|S_{m}\left(\alpha_{1}^{s}, \alpha_{2}^{s}, \ldots, \alpha_{n}^{s}\right)\right| & \leq \sum_{1 \leq s_{1}<\cdots<s_{m} \leq n}\left(q_{\lambda_{0}}^{\left(w_{s_{1}}(V)+\cdots+w_{s_{m}}(V)\right) / 2}\right)^{s} \\
& \leq \sum_{1 \leq s_{1}<\cdots<s_{m} \leq n}\left(q_{\lambda_{0}}^{\bar{w} / 2}\right)^{s}=\left(\begin{array}{c}
n \\
m
\end{array}\right)\left(q_{\lambda_{0}}^{\bar{w} / 2}\right)^{s} \leq c_{n} \ell_{0}^{d M u}
\end{aligned}
$$

and

$$
\begin{aligned}
\left|S_{m}\left(q_{\lambda_{0}}^{t_{1}}, q_{\lambda_{0}}^{t_{2}}, \ldots, q_{\lambda_{0}}^{t_{n}}\right)\right| & \leq \sum_{1 \leq s_{1}<\cdots<s_{m} \leq n} q_{\lambda_{0}}^{t_{s_{1}}+\cdots+t_{s_{m}}} \\
& \leq \sum_{1 \leq s_{1}<\cdots<s_{m} \leq n} q_{\lambda_{0}}^{n r u}=\left(\begin{array}{c}
n \\
m
\end{array}\right) q_{\lambda_{0}}^{n r u} \leq c_{n} \ell_{0}^{d M u}
\end{aligned}
$$

by (G-2), where $|\cdot|$ is the complex absolute value. Since we have $S_{m}\left(\alpha_{1}^{s}, \alpha_{2}^{s}, \ldots, \alpha_{n}^{s}\right) \equiv S_{m}\left(q_{\lambda_{0}}^{t_{1}}, q_{\lambda_{0}}^{t_{2}}, \ldots, q_{\lambda_{0}}^{t_{n}}\right)$ $\bmod \ell$ and $\ell>2 c_{n} \ell_{0}^{d M u}$, we obtain

$$
S_{m}\left(\alpha_{1}^{s}, \alpha_{2}^{s}, \ldots, \alpha_{n}^{s}\right)=S_{m}\left(q_{\lambda_{0}}^{t_{1}}, q_{\lambda_{0}}^{t_{2}}, \ldots, q_{\lambda_{0}}^{t_{n}}\right)
$$

for all $m$. This implies

$$
\begin{aligned}
\prod_{1 \leq k \leq n}\left(T-\alpha_{k}^{s}\right) & =\sum_{0 \leq m \leq n} S_{m}\left(\alpha_{1}^{s}, \alpha_{2}^{s}, \ldots, \alpha_{n}^{s}\right) T^{n-m} \\
& =\sum_{0 \leq m \leq n} S_{m}\left(q_{\lambda_{0}}^{t_{1}}, q_{\lambda_{0}}^{t_{2}}, \ldots, q_{\lambda_{0}}^{t_{n}}\right) T^{n-m} \\
& =\prod_{1 \leq k \leq n}\left(T-q_{\lambda_{0}}^{t_{k}}\right)
\end{aligned}
$$

and thus we finish the proof.

Now we start the proofs of Proposition 2.7 and 2.8. Take any representation $V$ which is an element of the set $\operatorname{Rep}_{\mathbb{Q}_{\ell}}\left(G_{K}\right)^{\circ}$ and denote its residual representation by $\bar{V}$. Then the representation $\bar{V}$ has a filtration of $G_{K}$-modules

$$
\{0\}=\bar{V}_{0} \subset \bar{V}_{1} \subset \cdots \subset \bar{V}_{n-1} \subset \bar{V}_{n}=\bar{V}
$$

such that $\bar{V}_{k}$ has dimension $k$ for each $1 \leq k \leq n$. We denote by $\psi_{k}: G_{K} \rightarrow \mathbb{F}_{\ell}^{\times}$the character corresponding to the action of $G_{K}$ on the quotient $\bar{V}_{k} / \bar{V}_{k-1}$ for each $1 \leq k \leq n$. Take any place $\lambda$ of $K$ above $\ell$. By Theorem 1.5, we obtain $\psi_{k}=\theta_{1, \lambda}^{b_{k, \lambda}}$ on $I_{\lambda}$ for some integer $0 \leq b_{k, \lambda} \leq e_{\lambda} r$, where $\theta_{1, \lambda}: I_{\lambda} \rightarrow \mathbb{F}_{\ell}^{\times}$is the fundamental character of level one at $\lambda$. Take a place $\lambda_{0}$ of $K$ above $\ell_{0}$ as in (G-2) and decompose $\operatorname{det}\left(T-\operatorname{Fr}_{\lambda_{0}} \mid V\right)=\prod_{k}\left(T-\alpha_{k}\right)$. Then, we see

$$
\left\{\alpha_{1}, \alpha_{2}, \ldots, \alpha_{n}\right\}=\left\{\psi_{1}\left(\operatorname{Fr}_{\lambda_{0}}\right), \psi_{2}\left(\operatorname{Fr}_{\lambda_{0}}\right), \ldots, \psi_{n}\left(\operatorname{Fr}_{\lambda_{0}}\right)\right\}
$$

in $\overline{\mathbb{F}}_{\ell}$. 
Proof of Proposition 2.7. Assume that $V$ is an element of the set $\operatorname{Rep}_{\mathbb{Q}_{\ell}}\left(G_{K}\right)_{\text {cycl }}^{\circ}$. Then we may suppose $\psi_{k}=\chi_{\ell}^{a_{k}}$ for any $k$ by (F-2). The relation $\chi_{\ell}^{a_{k}}=\theta_{1, \lambda}^{b_{k, \lambda}}$ on $I_{\lambda}$ implies $\theta_{1, \lambda}^{e_{\lambda} a_{k}}=\theta_{1, \lambda}^{b_{k, \lambda}}$ and thus $e_{\lambda} a_{k} \equiv b_{k, \lambda} \bmod \ell-1$. Hence we have $\chi_{\ell}^{e_{\lambda} a_{k}}=\chi_{\ell}^{b_{k, \lambda}}$ on $G_{K}$ and thus the set $\left\{\alpha_{1}^{e_{\lambda}}, \alpha_{2}^{e_{\lambda}}, \ldots, \alpha_{n}^{e_{\lambda}}\right\}$ coincides with the set $\left\{q_{\lambda_{0}}^{b_{1, \lambda}}, q_{\lambda_{0}}^{b_{2, \lambda}}, \ldots, q_{\lambda_{0}}^{b_{n, \lambda}}\right\}$ in $\overline{\mathbb{F}}_{\ell}$ by $(*)$. By Lemma 2.9, we have

$$
\left\{e_{\lambda} w_{1}(V) / 2, \ldots, e_{\lambda} w_{n}(V) / 2\right\}=\left\{b_{1, \lambda}, \ldots, b_{n, \lambda}\right\}
$$

if $\ell>2 B_{n} \ell_{0}^{d M e_{\lambda}}$. Since $e_{\lambda} \leq d$ and $e_{\lambda}=1$ if $\ell \nmid d_{K}$, we have the desired result.

Proof of Proposition 2.8. We note that each $\psi_{k}$ is unramified away from $\ell$ by (G-3). Now we assume that any one of the following conditions (A) or (B) holds:

(A) $\ell \nmid d_{K}$;

(B) No additional assumptions.

Setting $b_{k}^{\prime}:=b_{k, \lambda} / e_{\lambda} \in \mathbb{Q}$, we have $0 \leq b_{k}^{\prime} \leq r$. We note that, if we put

$$
D:= \begin{cases}1 & \text { under }(\mathrm{A}), \\ d & \text { under }(\mathrm{B}),\end{cases}
$$

then we see $D / e_{\lambda} \in \mathbb{Z}$. Since $\psi_{k}=\theta_{1, \lambda}^{b_{k, \lambda}}$ on $I_{\lambda}$, we see that $\psi_{k}^{e_{\lambda}} \chi_{\ell}^{-b_{k, \lambda}}$ is trivial on $I_{\lambda}$ and thus $\left(\psi_{k}^{e_{\lambda}} \chi_{\ell}^{-b_{k, \lambda}}\right)^{D / e_{\lambda}}=\psi_{k}^{D} \chi_{\ell}^{-b_{k}^{\prime} D}$ is also trivial on $I_{\lambda}$. Since the characters $\psi_{k}$ and $\chi_{\ell}$ are unramified away from $\ell$, this implies that $\psi_{k}^{D} \chi_{\ell}^{-b_{k}^{\prime} D}$ is unramified at all finite places of $K$ (recall that $\ell$ does not split in $K)$. By class field theory, it follows

$$
\psi_{k}^{D h_{K}^{+}}=\chi_{\ell}^{b_{k}^{\prime} D h_{K}^{+}}
$$

on $G_{K}$. Recall that $h_{K}^{+}$is the narrow class number of $K$. Thus we have that the set $\left\{\alpha_{1}^{D h_{K}^{+}}, \alpha_{2}^{D h_{K}^{+}}, \ldots, \alpha_{n}^{D h_{K}^{+}}\right\}$ coincides with the set $\left\{q_{\lambda_{0}}^{b_{1}^{\prime} D h_{K}^{+}}, q_{\lambda_{0}}^{b_{2}^{\prime} D h_{K}^{+}}, \ldots, q_{\lambda_{0}}^{b_{n}^{\prime} D h_{K}^{+}}\right\}$in $\overline{\mathbb{F}}_{\ell}$ by $(*)$. Now we assume $\ell>2 c_{n} \ell_{0}^{d D h_{K}^{+} M}$. Then we have

$$
\left\{D h_{K}^{+} w_{1}(V) / 2, \ldots, D h_{K}^{+} w_{n}(V) / 2\right\}=\left\{b_{1}^{\prime} D h_{K}^{+}, \ldots, b_{n}^{\prime} D h_{K}^{+}\right\}
$$

by Lemma 2.9. Our result comes from this equation.

Now we can obtain our main results.

Theorem 2.10. Suppose that $w$ is odd or $w>2 r$. Then the set $\operatorname{Re}_{\mathbb{Q}_{\ell}}\left(G_{K}\right)_{\text {cycl }}^{\bullet}$ is empty under any one of the following situations:

(a) $w$ is odd, $\ell \nmid d_{K}$ and $\ell>C_{1}$;

(b) $w$ is odd, the extension $K / \mathbb{Q}$ has odd degree and $\ell>C_{2}$;

(c) $w>2 r, \ell \nmid d_{K}$ and $\ell>C_{1}$;

(d) $w>2 r$ and $\ell>C_{2}$;

(e) $w$ and $n$ are odd, and $\ell>C_{2}$.

Theorem 2.11. Suppose that $w$ is odd or $w>2 r$. If $\ell$ does not split in $K$, then the $\operatorname{set} \operatorname{Rep}_{\mathbb{Q}_{\ell}}\left(G_{K}\right)^{\bullet}$ is empty under any one of the following situations:

(a) $w$ is odd, $\ell \nmid d_{K}$ and $\ell>C_{1}^{\prime}$;

(b) $w$ is odd, the extension $K / \mathbb{Q}$ has odd degree and $\ell>C_{2}^{\prime}$;

(c) $w>2 r, \ell \nmid d_{K}$ and $\ell>C_{1}^{\prime}$;

(d) $w>2 r$ and $\ell>C_{2}^{\prime}$;

(e) $w$ and $n$ are odd, and $\ell>C_{2}^{\prime}$. 
Proofs of Theorem 2.10 and 2.11. We only prove Theorem 2.10 because we can prove Theorem 2.11 by the same way. Suppose that there exists an $\ell$-adic Galois representation $V$ which is contained in $\operatorname{Rep}_{\mathbb{Q}_{\ell}}\left(G_{K}\right)_{\text {cycl }}^{\bullet}$ and take its residual representation $\bar{V}$. If we assume one of the situations (a) and (b) given in Proposition 2.7, then $\bar{V}$ is of uniform tame inertia weight $e_{\lambda} w / 2$ at any place $\lambda$ of $K$ above $\ell$, and thus $e_{\lambda} w / 2$ must be a rational integer. Moreover, by Theorem 1.5, it follows that the tame inertia weight $e_{\lambda} w / 2$ is between 0 and $e_{\lambda} r$. However, if we assume any one of the conditions (a), (b), (c) and (d), then $e_{\lambda} w$ is odd for some $\lambda$ or $e_{\lambda} w / 2>e_{\lambda} r$. This is a contradiction. The rest of the assertion related with (e) follows from the fact ([CS], Theorem 1) that the sum of all the tame inertia weights of $V$ at $\lambda$ must be divisible by $e_{\lambda}$.

Remark 2.12. To remove the special assumption " $\ell$ does not split in $K$ " in Theorem 2.11 is impossible in general because there exists such an example, which is pointed out by Akio Tamagawa: Let $E$ be an elliptic curves over $K$ with complex multiplication over $K$ by an imaginary quadratic field $F:=\mathbb{Q} \otimes_{\mathbb{Z}} \operatorname{End}_{K}(E) \subset K$. Then $E$ is potential everywhere good reduction and thus we may suppose $E$ has everywhere good reduction over $K$. Put $F_{\ell}:=\mathbb{Q}_{\ell} \otimes_{\mathbb{Q}} F$, which is a semisimple $\mathbb{Q}_{\ell}$-algebra. It is well-known that $F_{\ell}$ acts faithfully on the Tate-module $V_{\ell}(E)$ of $E$ and thus

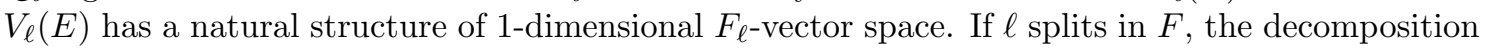
$F_{\ell} \simeq \mathbb{Q}_{\ell} \times \mathbb{Q}_{\ell}$ induces a decomposition of $V_{\ell}(E)$ as a sum of 1-dimensional $G_{K}$-stable $\ell$-adic representations. For such odd prime $\ell$, it is easy to check that $V_{\ell}(E)$ is an element of the set $\operatorname{Rep}_{\mathbb{Q}_{\ell}}\left(G_{K}\right)^{\bullet}$, where $\bullet=(2,2,1,1)$.

\section{Applications}

We give some applications of our results. We use same notation as in the previous section.

\subsection{Rasmussen-Tamagawa Conjecture}

As a first application, we show a special case of a Conjecture of Rasmussen and Tamagawa. We denote by $\tilde{K}_{\ell}$ the maximal pro- $\ell$ extension of $K\left(\mu_{\ell}\right)$ which is unramified away from $\ell$.

Definition 3.1. Let $g \geq 0$ be an integer. We denote by $\mathcal{A}(K, g, \ell)$ the set of $K$-isomorphism classes of abelian varieties $A$ over $K$, of dimension $g$, which satisfy the following equivalent conditions:

(1) $K\left(A\left[\ell^{\infty}\right]\right) \subset \tilde{K}_{\ell}$;

(2) The abelian variety $A$ has good reduction outside $\ell$ and the extension $K(A[\ell]) / K\left(\mu_{\ell}\right)$ is an $\ell$-extension;

(3) The abelian variety $A$ has good reduction outside $\ell$ and $A[\ell]$ admits a filtration of $G_{K}$-modules

$$
\{0\}=\bar{V}_{0} \subset \bar{V}_{1} \subset \cdots \subset \bar{V}_{2 g-1} \subset \bar{V}_{2 g}=A[\ell]
$$

such that $\bar{V}_{k}$ has dimension $k$ for each $1 \leq k \leq 2 g$. Furthermore, for each $1 \leq k \leq 2 g$, the $G_{K}$-action on the space $\bar{V}_{k} / \bar{V}_{k-1}$ is given by $g \cdot \bar{v}=\chi_{\ell}(g)^{a_{k}} \cdot \bar{v}$ for some $a_{k} \in \mathbb{Z}$.

The equivalently of the above three conditions follows from the criterion of Néron-Ogg-Shafarevich and Lemma 3.4 below (put $G=\operatorname{Gal}\left(K\left(A\left[\ell^{\infty}\right]\right) / K\right), N=\operatorname{Gal}\left(K\left(A\left[\ell^{\infty}\right]\right) / K\left(\mu_{\ell}\right)\right.$ ) and apply Lemma 3.4 to the group $A[\ell])$. The set $\mathcal{A}(K, g, \ell)$ is a finite set because of Faltings' proof of Shafarevich Conjecture. Rasmussen and Tamagawa conjectured that for any $\ell$ large enough, this set is empty:

Conjecture 3.2 ([RT], Conjecture 1). The set $\mathcal{A}(K, g):=\{(A, \ell) \mid[A] \in \mathcal{A}(K, g, \ell), \ell:$ prime number $\}$ is finite, that is, the set $\mathcal{A}(K, g, \ell)$ is empty for any prime $\ell$ large enough.

We call this conjecture the Rasmussen-Tamagawa conjecture. It is known that the RasmussenTamagawa conjecture holds under the following conditions:

(i) $K=\mathbb{Q}$ and $g=1([\mathrm{RT}]$, Theorem 2); 
(ii) $K$ is a quadratic number field other than the imaginary quadratic fields of class number one and $g=1$ ([RT], Theorem 4).

We consider the semistable reduction case of Conjecture 3.2.

Definition 3.3. (1) We denote by $\mathcal{A}(K, g, \ell)_{\text {st }}$ the set of $K$-isomorphism classes of abelian varieties in $\mathcal{A}(K, g, \ell)$ with everywhere semistable reduction.

(2) We denote by $\mathcal{A}\left(K, g, \ell_{0}, \ell\right)_{\text {st }}$ the set of $K$-isomorphism classes of abelian varieties $A$ over $K$ with everywhere semistable reduction, of dimension $g$, which satisfy the following condition: The abelian variety $A$ has good reduction at some places of $K$ above $\ell_{0}$ and $A[\ell]$ admits a filtration of $G_{K}$-modules

$$
\{0\}=\bar{V}_{0} \subset \bar{V}_{1} \subset \cdots \subset \bar{V}_{2 g-1} \subset \bar{V}_{2 g}=A[\ell]
$$

such that $\bar{V}_{k}$ has dimension $k$ for each $1 \leq k \leq 2 g$.

Clearly, we see $\mathcal{A}(K, g, \ell)_{\text {st }} \subset \mathcal{A}\left(K, g, \ell_{0}, \ell\right)_{\text {st }}$ since $\ell \neq \ell_{0}$. The set $\mathcal{A}(K, g, \ell)_{\text {st }}$ is finite, however, the set $\mathcal{A}\left(K, g, \ell_{0}, \ell\right)_{\text {st }}$ may be infinite. The Rasmussen-Tamagawa conjecture implies that $\mathcal{A}(K, g, \ell)_{\text {st }}$ will be empty for a prime $\ell$ large enough. We will prove that $\mathcal{A}\left(K, g, \ell_{0}, \ell\right)_{\text {st }}$ is in fact empty for a prime $\ell$ large enough which does not split in $K$. Recall the lemma proved by Rasmussen and Tamagawa (cf. [RT], Lemma 3 ). Let $G$ be a topological group with a normal pro- $\ell$ open subgroup $N$, such that the quotient $\Delta=G / N$ is isomorphic to a subgroup of $\mathbb{F}_{\ell}^{\times}$. Because $N$ is pro- $\ell$, we see that $N$ has trivial image under any character $\psi: G \rightarrow \mathbb{F}_{\ell}^{\times}$. Hence, there always exists an induced character $\bar{\psi}: \Delta \rightarrow \mathbb{F}_{\ell}^{\times}$. Let $\chi: G \rightarrow \mathbb{F}_{\ell}^{\times}$be a character such that the induced character $\bar{\chi}$ is an injection $\Delta \hookrightarrow \mathbb{F}_{\ell}^{\times}$. Finally, let $\bar{V}$ be a finite dimensional $\mathbb{F}_{\ell}$-vector space of dimension $n$ on which $G$ acts continuously.

Lemma 3.4. The vector space $V$ admits a filtration of $G_{K}$-modules

$$
\{0\}=\bar{V}_{0} \subset \bar{V}_{1} \subset \cdots \subset \bar{V}_{n-1} \subset \bar{V}_{n}=\bar{V}
$$

such that $\bar{V}_{k}$ has dimension $k$ for each $1 \leq k \leq n$. Furthermore, for each $1 \leq k \leq n$, the $G$-action on the space $\bar{V}_{k} / \bar{V}_{k-1}$ is given by $g . \bar{v}=\chi(g)^{a_{k}} \cdot \bar{v}$ for some $a_{k} \in \mathbb{Z}, 0 \leq a_{k}<\# \Delta$.

Proof. The proof will proceed by the same method as the proof of Lemma 3 of [RT], thus we omit it.

Take an abelian variety $A$ which is in the set $\mathcal{A}(K, g, \ell)_{\text {st }}\left(\operatorname{resp} . \mathcal{A}\left(K, g, \ell_{0}, \ell\right)_{\text {st }}\right)$. Then $V_{\ell}(A)$ is an element of the set $\operatorname{Rep}_{\mathbb{Q}_{\ell}}\left(G_{K}\right)_{\text {cycl }}^{\bullet}\left(\operatorname{resp} \operatorname{Rep}_{\mathbb{Q}_{\ell}}\left(G_{K}\right)^{\bullet}\right)$ with $\bullet=(2 g, 2,1,1)\left(\operatorname{resp} \cdot \bullet=\left(2 g, \ell_{0}, 1,1\right)\right)$ for any $\ell>2$ (resp. $\left.\ell>\ell_{0}\right)$. Consequently, we obtain the following results as corollaries of Theorem 2.10 and 2.11:

Corollary 3.5. The set $\mathcal{A}(K, g, \ell)_{\text {st }}$ is empty under any one of the following situations:

(a) $\ell \nmid d_{K}$ and $\ell>2^{\delta_{1}}\left(\begin{array}{c}2 g \\ g\end{array}\right)$, where $\delta_{1}:=2 d g+1$;

(b) The extension $K / \mathbb{Q}$ has odd degree and $\ell>2^{\delta_{2}}\left(\begin{array}{c}2 g \\ g\end{array}\right)$, where $\delta_{2}:=2 d^{2} g+1$.

Corollary 3.6. Suppose that $\ell$ does not split in $K$. The set $\mathcal{A}\left(K, g, \ell_{0}, \ell\right)_{\mathrm{st}}$ is empty under any one of the following situations:

(a) $\ell \nmid d_{K}$ and $\ell>2 \ell_{0}^{\delta_{1}^{\prime}}\left(\begin{array}{c}2 g \\ g\end{array}\right)$, where $\delta_{1}^{\prime}:=2 d g h_{K}^{+}$;

(b) The extension $K / \mathbb{Q}$ has odd degree and $\ell>2 \ell_{0}^{\delta_{2}^{\prime}}\left(\begin{array}{c}2 g \\ g\end{array}\right)$, where $\delta_{2}^{\prime}:=2 d^{2} g h_{K}^{+}$.

Remark 3.7. Rasmussen and Tamagawa have shown the finiteness of the set $\mathcal{A}(K, g)_{\text {st }}$ by using the result of $[\mathrm{Ra}]$ instead of Theorem 1.5 (unpublished). Our main results in this paper are motivated by their work. 


\subsection{Irreducibility of $\ell$-torsion points of elliptic curves}

We consider the following classical question:

Question 3.8. Does there exist a constant $c_{K}$, which depends only on $K$, such that for any semistable elliptic curve $E$ defined over $K$ without complex multiplication over $K$, the representation in its $\ell$-torsion points $E[\ell]$ is irreducible whenever $\ell>c_{K}$ ? Furthermore, if the answer is positive, how can we evaluate such a constant $c_{K}$ ?

By Mazur's results on a moduli of rational points of modular curve $X_{0}(N)([\mathrm{Ma}])$, it is known that $c_{\mathbb{Q}}=7$. If $K$ is a quadratic field, then the existence of $c_{K}$ is known and moreover, if the class number of $K$ is 1 , then the explicit calculation of $c_{K}$ is given by Kraus [Kr1]. By combining results on Merel $([\mathrm{Me}])$ and Momose $([\mathrm{Mo}])$, Kraus showed the existence of $c_{K}$ for a number field $K$ which does not contain an imaginary quadratic field of class number $1([\mathrm{Kr} 2])$. Moreover, Kraus defined the good condition "(C)" associated with $K$ in op. cit, such that the existence and the explicit value of $c_{K}$ is known if $K$ satisfies this condition.

The following is easy consequence of Corollary 3.6 under the case $g=1$.

Corollary 3.9. Let $E$ be an elliptic curve over $K$ with everywhere semistable reduction. Let $\ell_{E}$ be the minimal prime number $p$ such that $E$ has good reduction at some finite places of $K$ above $p$. Suppose $\ell$ does not split in $K$. Then $E[\ell]$ is irreducible under any one of the following conditions:

(a) $\ell \nmid d_{K}$ and $\ell>4 \ell_{E}^{\delta_{1}^{\prime \prime}}$, where $\delta_{1}^{\prime \prime}:=2 d h_{K}^{+}$;

(b) The extension $K / \mathbb{Q}$ has odd degree and $\ell>4 \ell_{E}^{\delta_{2}^{\prime \prime}}$, where $\delta_{2}^{\prime \prime}:=2 d^{2} h_{K}^{+}$.

We remark that the above corollary is valid even if $E$ has complex multiplication over $K$.

\subsection{Residual properties of étale cohomology groups}

For any semistable elliptic curve $E$ over $\mathbb{Q}$, Serre proved the following ([Se], Section 5.4, Proposition 21, Corollary 1): Let $\ell_{E}$ be the minimal prime number $p$ such that $E$ has good reduction at $p$. Then $E[\ell]$ is irreducible if $\ell>\left(1+\ell_{E}^{1 / 2}\right)^{2}$.

As a corollary of Theorem 2.11, we can slightly generalize this fact to étale cohomology groups of odd degree.

Corollary 3.10. Let $X$ be a proper smooth scheme over $K$ with everywhere semistable reduction and $w$ an odd integer. Let $b_{w}(X)$ be a $w$-th Betti number of $X$ and $\ell_{X}$ the minimal prime number $p$ such that $X$ has good reduction at some places of $K$ above $p$. Then there exists a constant $C$ depending only on $b_{w}(X)$ and $\ell_{X}$ such that for any prime number $\ell>C$ which does not split in $K$, the étale cohomology group $H_{\mathrm{ét}}^{w}\left(X_{\bar{K}}, \mathbb{Q}_{\ell}\right)$ is not of residually Borel. More precisely, if $\ell$ does not split in $K, H_{\mathrm{ét}}^{w}\left(X_{\bar{K}}, \mathbb{Q}_{\ell}\right)$ is not of residually Borel under any one of the following conditions:

(a) $\ell \nmid d_{K}$ and $\ell>2 B_{b_{w}(X)} \ell_{X}^{\Delta_{1}}$, where $\Delta_{1}:=b_{w}(X) d h_{K}^{+} w$;

(b) The extension $K / \mathbb{Q}$ has odd degree and $\ell>2 B_{b_{w}(X)} \ell_{X}^{\Delta_{2}}$, where $\Delta_{2}:=b_{w}(X) d^{2} h_{K}^{+} w$.

Proof. Putting $\bullet:=\left(b_{w}(X), \ell_{X}, w, w\right)$, we see that the dual of $H_{\mathrm{e} t}^{w}\left(X_{\bar{K}}, \mathbb{Q}_{\ell}\right)$ is contained in the set $\operatorname{Rep}_{\mathbb{Q}_{\ell}}\left(G_{K}\right)^{\bullet}$. Applying Theorem 2.11, we obtain the desired result.

For any proper smooth scheme $X$ over $K$, there exists an finite extension $L$ over $K$ such that $H_{\text {ét }}^{w}\left(X_{\bar{L}}, \mathbb{Q}_{\ell}\right)$ is everywhere semistable as a representation of $G_{L}$ for almost all $\ell$ by Proposition 2.3. If this is the case, we see that $H_{\text {ét }}^{w}\left(X_{\bar{L}}, \mathbb{Q}_{\ell}\right)^{\vee}$ satisfies $(\mathrm{G}-1),(\mathrm{G}-2)$ and $(\mathrm{G}-3)$ as a representation of $G_{L}$. Thus if we can obtain the explicit description of $L$, we will able to obtain the analogous result of corollary 3.10 for a prime $\ell$ large enough which does not split in $L$. However, it is very difficult to determine such $L$ in general. We can determine this $L$ if $X$ is an abelian variety. If this is the case, Raynaud's criterion of semistable reduction ([Gr], Proposition 4.7) implies that $X$ is everywhere semistable reduction over $L:=K(X[3], X[5])$. 


\section{References}

[Ca] Xavier Caruso, Représentations semi-stables de torsion dans le case er $<p-1$, J. Reine Angew. Math. 594, 35-92 (2006).

[CS] Xavier Caruso and David Savitt, Polygons de Hodge, de Newton et de línertie moderee des representations semi-stables, Math. Ann. 343, 773-789 (2009).

[dJ] Aise Johan de Jong, Smoothness, semi-stability and alterations, Publ. Math. IHES 83, 51-93 (1996).

[Fo] Jean-Marc Fontaine, Modules galoisiennes, modules filtrés et anneaux de Barsotti-Tate, Journées de Géométrie Algébrique de Rennes, vol III, Astérisque 65, Soc. Math. de France, Paris, 3-80 (1979).

[Gr] Alexander Grothendieck, Modèles de Néron et monodromie, in Groupes de monodromie en géometrie algébrique, SGA 7, Lecture Notes in Mathematics, vol. 288, 313-523 (1972).

[Kr1] Alain Kraus, Courbes elliptiques semi-stables et corps quadratiques, J. Number Theory 60, 245-253 (1996).

[Kr2] Alain Kraus, Courbes elliptiques semi-stables sur les corps de nombres, Int. J. Number Theory 3, 611-633 (2007).

[La] Serge Lang, Algebraic Number Theory, Second Edition, Graduate Texts in Mathematics, vol. 110, Springer, New York, (1994).

[Ma] Barry Mazur, Rational isogenies of prime degree, Invent. Math. 44, 129-162 (1978).

[Me] Loïc Merel, Bornes pour la torsion des courbes elliptiques sur les corps de nombres, Invent. Math. 124, 437-449 (1996).

[Mo] Fumiyuki Momose, Isogenies of prime degree over number fields, Compositio Math. 97, 329348 (1995).

[RT] Christopher Rasmussen and Akio Tamagawa, A finiteness conjecture on abelian varieties with constrained prime power torsion, Math. Res. Lett. 15, 1223-1231 (2008).

[Ra] Michel Raynaud, Schémas en groupes de type $(p, \ldots, p)$, Bulletin de la Société Mathématique de France 102, 241-280 (1974).

[Se] Jean-Pierre Serre, Propriétés galoisiennes des points d'ordre fini des courbes elliptiques, Invent. Math. 4, 259-331 (1972).

[Ts] Takeshi Tsuji, Semi-stable conjecture of Fontaine-Jannsen: A survey, Astérisque. 279, 323370 (2002). 


\section{List of MI Preprint Series, Kyushu University}

The Global COE Program

Math-for-Industry Education \& Research Hub

MI

MI2008-1 Takahiro ITO, Shuichi INOKUCHI \& Yoshihiro MIZOGUCHI

Abstract collision systems simulated by cellular automata

MI2008-2 Eiji ONODERA

The intial value problem for a third-order dispersive flow into compact almost

Hermitian manifolds

MI2008-3 Hiroaki KIDO

On isosceles sets in the 4-dimensional Euclidean space

MI2008-4 Hirofumi NOTSU

Numerical computations of cavity flow problems by a pressure stabilized characteristiccurve finite element scheme

MI2008-5 Yoshiyasu OZEKI

Torsion points of abelian varieties with values in nfinite extensions over a padic field

MI2008-6 Yoshiyuki TOMIYAMA

Lifting Galois representations over arbitrary number fields

MI2008-7 Takehiro HIROTSU \& Setsuo TANIGUCHI

The random walk model revisited

MI2008-8 Silvia GANDY, Masaaki KANNO, Hirokazu ANAI \& Kazuhiro YOKOYAMA Optimizing a particular real root of a polynomial by a special cylindrical algebraic decomposition

MI2008-9 Kazufumi KIMOTO, Sho MATSUMOTO \& Masato WAKAYAMA

Alpha-determinant cyclic modules and Jacobi polynomials 
MI2008-10 Sangyeol LEE \& Hiroki MASUDA

Jarque-Bera Normality Test for the Driving Lévy Process of a Discretely Observed Univariate SDE

MI2008-11 Hiroyuki CHIHARA \& Eiji ONODERA

A third order dispersive flow for closed curves into almost Hermitian manifolds

MI2008-12 Takehiko KINOSHITA, Kouji HASHIMOTO and Mitsuhiro T. NAKAO

On the $L^{2}$ a priori error estimates to the finite element solution of elliptic problems with singular adjoint operator

MI2008-13 Jacques FARAUT and Masato WAKAYAMA

Hermitian symmetric spaces of tube type and multivariate Meixner-Pollaczek polynomials

MI2008-14 Takashi NAKAMURA

Riemann zeta-values, Euler polynomials and the best constant of Sobolev inequality

MI2008-15 Takashi NAKAMURA

Some topics related to Hurwitz-Lerch zeta functions

MI2009-1 Yasuhide FUKUMOTO

Global time evolution of viscous vortex rings

MI2009-2 Hidetoshi MATSUI \& Sadanori KONISHI

Regularized functional regression modeling for functional response and predictors

MI2009-3 Hidetoshi MATSUI \& Sadanori KONISHI

Variable selection for functional regression model via the $L_{1}$ regularization

MI2009-4 Shuichi KAWANO \& Sadanori KONISHI

Nonlinear logistic discrimination via regularized Gaussian basis expansions

MI2009-5 Toshiro HIRANOUCHI \& Yuichiro TAGUCHII

Flat modules and Groebner bases over truncated discrete valuation rings 
MI2009-6 Kenji KAJIWARA \& Yasuhiro OHTA

Bilinearization and Casorati determinant solutions to non-autonomous $1+1$ dimensional discrete soliton equations

\section{MI2009-7 Yoshiyuki KAGEI}

Asymptotic behavior of solutions of the compressible Navier-Stokes equation around the plane Couette flow

MI2009-8 Shohei TATEISHI, Hidetoshi MATSUI \& Sadanori KONISHI

Nonlinear regression modeling via the lasso-type regularization

MI2009-9 Takeshi TAKAISHI \& Masato KIMURA

Phase field model for mode III crack growth in two dimensional elasticity

MI2009-10 Shingo SAITO

Generalisation of Mack's formula for claims reserving with arbitrary exponents for the variance assumption

MI2009-11 Kenji KAJIWARA, Masanobu KANEKO, Atsushi NOBE \& Teruhisa TSUDA Ultradiscretization of a solvable two-dimensional chaotic map associated with the Hesse cubic curve

\section{MI2009-12 Tetsu MASUDA}

Hypergeometric T -functions of the q-Painlevé system of type $E_{8}^{(1)}$

MI2009-13 Hidenao IWANE, Hitoshi YANAMI, Hirokazu ANAI \& Kazuhiro YOKOYAMA A Practical Implementation of a Symbolic-Numeric Cylindrical Algebraic Decomposition for Quantifier Elimination

MI2009-14 Yasunori MAEKAWA

On Gaussian decay estimates of solutions to some linear elliptic equations and its applications

MI2009-15 Yuya ISHIHARA \& Yoshiyuki KAGEI

Large time behavior of the semigroup on $L^{p}$ spaces associated with the linearized compressible Navier-Stokes equation in a cylindrical domain 
MI2009-16 Chikashi ARITA, Atsuo KUNIBA, Kazumitsu SAKAI \& Tsuyoshi SAWABE Spectrum in multi-species asymmetric simple exclusion process on a ring

MI2009-17 Masato WAKAYAMA \& Keitaro YAMAMOTO

Non-linear algebraic differential equations satisfied by certain family of elliptic functions

MI2009-18 Me Me NAING \& Yasuhide FUKUMOTO

Local Instability of an Elliptical Flow Subjected to a Coriolis Force

MI2009-19 Mitsunori KAYANO \& Sadanori KONISHI

Sparse functional principal component analysis via regularized basis expansions and its application

MI2009-20 Shuichi KAWANO \& Sadanori KONISHI

Semi-supervised logistic discrimination via regularized Gaussian basis expansions

MI2009-21 Hiroshi YOSHIDA, Yoshihiro MIWA \& Masanobu KANEKO

Elliptic curves and Fibonacci numbers arising from Lindenmayer system with symbolic computations

MI2009-22 Eiji ONODERA

A remark on the global existence of a third order dispersive flow into locally Hermitian symmetric spaces

MI2009-23 Stjepan LUGOMER \& Yasuhide FUKUMOTO

Generation of ribbons, helicoids and complex scherk surface in laser-matter Interactions

MI2009-24 Yu KAWAKAMI

Recent progress in value distribution of the hyperbolic Gauss map

MI2009-25 Takehiko KINOSHITA \& Mitsuhiro T. NAKAO

On very accurate enclosure of the optimal constant in the a priori error estimates for $H_{0}^{2}$-projection 
MI2009-26 Manabu YOSHIDA

Ramification of local fields and Fontaine's property (Pm)

MI2009-27 Yu KAWAKAMI

Value distribution of the hyperbolic Gauss maps for flat fronts in hyperbolic three-space

MI2009-28 Masahisa TABATA

Numerical simulation of fluid movement in an hourglass by an energy-stable finite element scheme

MI2009-29 Yoshiyuki KAGEI \& Yasunori MAEKAWA Asymptotic behaviors of solutions to evolution equations in the presence of translation and scaling invariance

MI2009-30 Yoshiyuki KAGEI \& Yasunori MAEKAWA

On asymptotic behaviors of solutions to parabolic systems modelling chemotaxis

MI2009-31 Masato WAKAYAMA \& Yoshinori YAMASAKI

Hecke's zeros and higher depth determinants

MI2009-32 Olivier PIRONNEAU \& Masahisa TABATA

Stability and convergence of a Galerkin-characteristics finite element scheme of lumped mass type

MI2009-33 Chikashi ARITA

Queueing process with excluded-volume effect

MI2009-34 Kenji KAJIWARA, Nobutaka NAKAZONO \& Teruhisa TSUDA

Projective reduction of the discrete Painlevé system of type $\left(A_{2}+A_{1}\right)^{(1)}$

MI2009-35 Yosuke MIZUYAMA, Takamasa SHINDE, Masahisa TABATA \& Daisuke TAGAMI Finite element computation for scattering problems of micro-hologram using DtN map 
MI2009-36 Reiichiro KAWAI \& Hiroki MASUDA

Exact simulation of finite variation tempered stable Ornstein-Uhlenbeck processes

MI2009-37 Hiroki MASUDA

On statistical aspects in calibrating a geometric skewed stable asset price model

MI2010-1 Hiroki MASUDA

Approximate self-weighted LAD estimation of discretely observed ergodic OrnsteinUhlenbeck processes

MI2010-2 Reiichiro KAWAI \& Hiroki MASUDA

Infinite variation tempered stable Ornstein-Uhlenbeck processes with discrete observations

MI2010-3 Kei HIROSE, Shuichi KAWANO, Daisuke MIIKE \& Sadanori KONISHI Hyper-parameter selection in Bayesian structural equation models

MI2010-4 Nobuyuki IKEDA \& Setsuo TANIGUCHI

The Itô-Nisio theorem, quadratic Wiener functionals, and 1-solitons

MI2010-5 Shohei TATEISHI \& Sadanori KONISHI

Nonlinear regression modeling and detecting change point via the relevance vector machine

MI2010-6 Shuichi KAWANO, Toshihiro MISUMI \& Sadanori KONISHI

Semi-supervised logistic discrimination via graph-based regularization

MI2010-7 Teruhisa TSUDA

UC hierarchy and monodromy preserving deformation

MI2010-8 Takahiro ITO

Abstract collision systems on groups

MI2010-9 Hiroshi YOSHIDA, Kinji KIMURA, Naoki YOSHIDA, Junko TANAKA \& Yoshihiro MIWA

An algebraic approach to underdetermined experiments 
MI2010-10 Kei HIROSE \& Sadanori KONISHI

Variable selection via the grouped weighted lasso for factor analysis models

MI2010-11 Katsusuke NABESHIMA \& Hiroshi YOSHIDA

Derivation of specific conditions with Comprehensive Groebner Systems

MI2010-12 Yoshiyuki KAGEI, Yu NAGAFUCHI \& Takeshi SUDOU

Decay estimates on solutions of the linearized compressible Navier-Stokes equation around a Poiseuille type flow

MI2010-13 Reiichiro KAWAI \& Hiroki MASUDA

On simulation of tempered stable random variates

MI2010-14 Yoshiyasu OZEKI

Non-existence of certain Galois representations with a uniform tame inertia weight 